\title{
Padrões de acessos a informações sobre proteção antiUV durante os verões brasileiros: haveria um "efeito verão"?
}

\author{
Patterns of access to information on protection against UV during \\ the Brazilian summer: is there such a thing as the "summer effect"?
}

Paulo Roberto Vasconcellos-Silva ${ }^{1,2}$

Rosane Harter Griep ${ }^{2}$

Miriam Carvalho de Souza ${ }^{3}$

${ }^{1}$ Coordenadoria de Pesquisa, Instituto Nacional do Câncer (INCA). Pça. Cruz Vermelha 23, Centro 20230-130 Rio de Janeiro RJ Brasil.

pr@iocc.fiocruz.br

${ }^{2}$ Escola Nacional de Saúde Pública Sergio Arouca,

Fiocruz.

${ }^{3}$ Coordenação de Prevenção

e Vigilância, INCA.

\begin{abstract}
Internet search patterns associated with "windows" of collective interest have been increasingly investigated in the field of public health. This article sets out to identify search patterns relating to the quest for information on skin protection after the perception of excessive exposure to $U V$ radiation - the so-called "summer effect" as it is commonly referred to in Brazil. To calculate the number of hits on the Brazilian National Cancer Institute website - a renowned source of information resources on prevention - log analyzer software was used to measure the volume of hits on specific content pages. The pages on skin protection and self-examination (pages of interest) were monitored over a 48-month period. It was seen that, although the monthly average of hits on pages of interest revealed statistically significant annual growth, the results for the analysis of variance showed no significant differences between the number of hits in the summer compared with other months ( $p=0.7491)$. In short, the perception of intense exposure to the summer sun did not encourage further interest to search for information on prevention.
\end{abstract}

Key words Internet and health, Mass communication, Skin cancer, Primary prevention, Mass media
Resumo Padrões de buscas na internet (queries) vinculados a "janelas" de interesses coletivos têm sido objeto de crescente investigação no campo da saúde pública. O presente artigo pretende identificar padrões de queries acerca de informações sobre a proteção da pele perante a exposição excessiva à radiação UV - o que aqui chamamos de "efeito verão". Para estimar os acessos ao site do Instituto Nacional de Câncer - notória fonte de recursos informativos sobre prevenção - foi usado um software (Log analyzer) que mensura o volume de acessos a conteúdos específicos. Durante 48 meses foram acompanhadas as páginas sobre proteção da pele e autoexame (páginas de interesse). Observou-se que, embora a média de acessos tenha crescido significativamente, os resultados da análise de variância não mostraram diferenças significativas entre os realizados no verão e os feitos nos demais meses ( $p=0,7491)$. A percepção da intensa exposição ao sol de verão não incentivou interesse suplementar por buscas sobre prevenção. Discute-se se a dissonância entre o conhecimento individual acerca do próprio estado de saúde em relação às medidas ligadas à sua preservação, pois talvez exerçam "influências antiprevenção" sobre o interesse coletivo a respeito da proteção da saúde. Palavras-chave Internet e saúde, Câncer de pele, Prevenção primária, Meios de comunicação em massa 


\section{Introdução}

As campanhas para diagnóstico precoce e prevenção do câncer de pele têm se apresentado como um desafio aos planejadores nos países mais expostos à radiação ultravioleta (UV). As frequentes campanhas australianas resultaram em estabilização na incidência de novos casos de carcinomas basocelulares e melanomas entre pessoas a partir da sexta década de vida ${ }^{1}$. No entanto, entre a população mais jovem - usualmente usuários mais assíduos da internet - o controle permanece como desafio e prioridade para os sistemas de saúde ${ }^{2}$. A partir dos anos 1970 e 1980 foi possível identificar expressivos aumentos anuais na incidência de novos casos de melanomas (3-10\%) em populações caucasianas, o que suscitou uma intensificação das campanhas de prevenção/proteção da pele, assim como o autoexame da pele $(\mathrm{AEP})^{3,4}$. Percebemos que as campanhas de prevenção e identificação precoce do câncer parecem ter alcance limitado a depender do que se deseja prevenir na perspectiva de determinadas circunstâncias socioculturais ${ }^{5}$. Nesse contexto, estudos voltados à avaliação de elementos culturais que poderiam incentivar a busca por informações para prevenção do câncer ainda são inexistentes em nosso país. A pesquisa sobre o uso coletivo da internet direcionada à proteção da saúde e as tendências de buscas (ou queries) como padrões de acessos a indicar demandas coletivas ainda estão por se consolidar no Brasil, embora se tornem cada vez mais empregadas no exterior. A identificação de queries tem sido objeto de crescente investigação como meio para avaliar o interesse coletivo voltado a determinados conteúdos ${ }^{6}$, sobretudo àqueles ligados às matérias de saúde pública. Pesquisadores do Google, estudando queries georeferenciadas, puderam identificar precocemente surtos de influenza por intermédio das tendências daquelas ligadas a sintomas típicos da doença ${ }^{7}$, e $\mathrm{Ocampo}^{8}$ descreveu recentemente focos endêmicos de malária na Tailândia pelos mesmos caminhos. Padrões de queries também já revelaram efeitos colaterais de medicamentos ${ }^{9}$ e surtos de gastroenterite aguda ${ }^{10}$. Não obstante, poucos estudos têm se dedicado a retratar o interesse social a conteúdos ligados à prevenção primária $\mathrm{e}$ secundária de doenças e agravos, o que poderia subsidiar o planejamento de intervenções institucionais mais estimulantes àqueles que buscam por fontes qualificadas. Nesse contexto, o presente artigo é parte de um projeto mais abrangente voltado à identificação de padrões de acessos a conteúdos sobre a prevenção do câncer. Busca-se identificar e retratar oscilações das buscas coletivas voltadas à prevenção das neoplasias para saber se há "janelas de interesse" que poderiam ser oportunamente aproveitadas. Entende-se que o intenso fluxo e a exacerbada competição de dados ao qual nos submetemos diariamente talvez deixem poucos espaços para as mensagens institucionais que parecem ter se padronizado ao longo dos anos. No caso em questão, objetivou-se caracterizar "janelas de interesse" equivalentes à intensificação de padrões de acessos a informações sobre prevenção do câncer de pele, retratando um período de acelerada popularização da internet no Brasil. Para tanto, considerou-se a seguinte pergunta de pesquisa - a percepção da intensa exposição aos raios solares durante os meses de verão, assim como os frequentes incidentes ligados à superexposição peculiares à época, incentivariam a busca por dados relacionados à proteção ao UV e ao AEP? A percepção de exposição excessiva influenciaria as pessoas a buscar informações sobre a proteção da pele - que aqui chamamos de "efeito verão"? O sítio escolhido para a coleta de dados (www.inca.gov.br) é notória fonte de recursos informativos sobre a prevenção do câncer, o que se evidencia por seu posicionamento destacado nas primeiras inserções do Google quando se usa termos de busca como "câncer de pele"; "prevenção do câncer"; "autoexame de pele"; entre outras combinações assemelhadas.

\section{Metodologia}

Ao contrário das estimativas baseadas em buscadores genéricos (como o Google ou o Bing!, que lidam com conteúdos de qualquer origem), o estudo de queries a partir de websites institucionais consagrados como fontes de comunicação entre experts e o público geral oferecem um discurso institucional público puro, sem as controvérsias técnicas peculiares à comunicação interpares. Os conteúdos usualmente são confiáveis e é possível alcançar um sofisticado nível de detalhamento no que se refere ao acesso a conteúdos ligados à prevenção primária, à identificação precoce ou às tecnologias de diagnósticos ou terapêuticas. É, assim, possível delinear o foco e o nível de interesse das buscas mais focadas e persistentes em determinados assuntos que talvez possam indicar o sentido de uma percepção na direção de eventuais decisões em saúde. A escolha do website do Instituto Nacional de Câncer (INCA) como campo de coleta de dados se justifica em vista de seu posicionamento destacado no page rank do bus- 
cador Google nos assuntos ligados à matéria. As páginas de interesse apresentam conteúdos detalhados e atualizados sobre a proteção antirradiação UV, assim como instruções ilustradas sobre o AEP, com descrições, fotos e possibilidade de download de material informativo.

Para avaliação dos acessos às páginas do INCA foi usado um software (Log analyzer da Webtrends, versão 5.0) que emite relatórios em planilhas mensais através das quais é possível mensurar o volume de acessos a páginas específicas do site em termos de médias mensais. Além destas, os acessos também podem ser registrados como "tempo médio de permanência nas páginas", "percentual de acessos a páginas" em relação ao website e "razão de retorno mensal" - razão do número de acessos pelo número de usuários a cada mês. Optamos por utilizar somente a média mensal de acessos às páginas para simplificar a exposição dos resultados e cientes de que esses indicadores usualmente oscilam em mútua consistência ${ }^{5,11}$. As páginas de interesse foram acompanhadas ao longo de 48 meses - de 2004 a 2009 - período de popularização da internet no Brasil e expressivo crescimento de acessos às páginas do INCA. Além disso, a partir de 2010 os conteúdos do website do INCA foram redimensionados e reagrupados, o que poderia acrescentar confundimento à aferição dos desfechos . Os meses nos quais se observa a maior incidência de irradiação UV, assim como as maiores sensações térmicas (de Dezembro a Março no hemisfério Sul) foram comparados às médias anuais por meio de Análises de Variância (ANOVA) admitindo um nível de confiança de 95\%. Esse procedimento estatístico permite a comparação simultânea de diferentes médias (médias anuais $\mathrm{x}$ médias de Dezembro a Março) visando determinar, por meio de um teste $\mathrm{F}$, se as diferenças observadas são estatisticamente significativas ou não.

\section{Resultados}

O acompanhamento do número médio de acessos às páginas de interesse e seus respectivos intervalos de confiança revelou um crescimento anual estatisticamente significativo entre 2006 e 2009 (Gráfico 1 e Tabela 1).

A análise dos valores médios e dos intervalos de confiança (Tabela 1) indica que a média de acessos às páginas de interesse nos quatro períodos de maior incidência de irradiação UV não foi superior às respectivas médias anuais ao longo dos 4 anos. Os resultados da análise de variância não demonstram diferenças estatisticamente significativas entre o número de acessos no verão e nos demais meses $(p=0,7491)$ (Tabela 1$)$.

\section{Discussão e conclusões}

Pesquisas acerca das necessidades de informação em saúde identificadas por meio do estudo de padrões de acessos à Internet têm se intensificado no meio acadêmico internacional nos últimos anos. A compreensão da origem e o sentido atribuído às necessidades de uma sociedade que se vale cada vez mais da internet, sob determinadas circunstâncias, é atualmente percebida como fator essencial ao aprimoramento das estruturas assistenciais e das campanhas de esclarecimento coletivo. Não obstante, há poucos estudos dedicados a tais padrões de acessos em países socioeconomicamente desfavorecidos, nos quais se observa um aumento exponencial na apropriação social das novas tecnologias de comunicação. Em pesquisas anteriores ${ }^{11}$, descrevemos o desenvolvimento de determinados círculos de atenção influenciados por eventos ostensivamente divulgados pelas mídias e ligados ao adoecimento de figuras públicas e personagens de ficção. Com base nessa lógica, levantou-se a hipótese de que a percepção coletiva de calor e a superexposição à irradiação solar decorrente do verão brasileiro, poderia se associar à procura por informações ligadas à proteção e ao autoexame da pele. Seria razoável supor que nos meses mais quentes e de maior exposição aos raios UV, haveria interesse suplementar nos conteúdos que esclarecessem sobre como preservar a pele dos malefícios da irradiação solar excessiva.

Contrariando nossas hipóteses, não foram encontradas diferenças estatisticamente significativas no volume de acessos às páginas de interesse durante o verão, quando se observa uma frequência aumentada aos acidentes relacionados à superexposição solar ${ }^{12}$. Pelos meios aqui descritos, não foi possível caracterizar um "efeito verão" no território nacional. Apesar de ser possível identificar o crescimento do interesse coletivo voltado às páginas do INCA - consistente com o aumento do recurso à internet como suporte ao autocuidado ${ }^{13}$ - a percepção da intensa exposição ao sol de verão não incentivou o interesse às buscas às páginas sobre prevenção e AEP. Achados semelhantes foram descritos há duas décadas ${ }^{14}$, quando o acesso à internet ainda não havia se popularizado, embora já houvesse a noção coletiva de dano à pele pela superexposição 


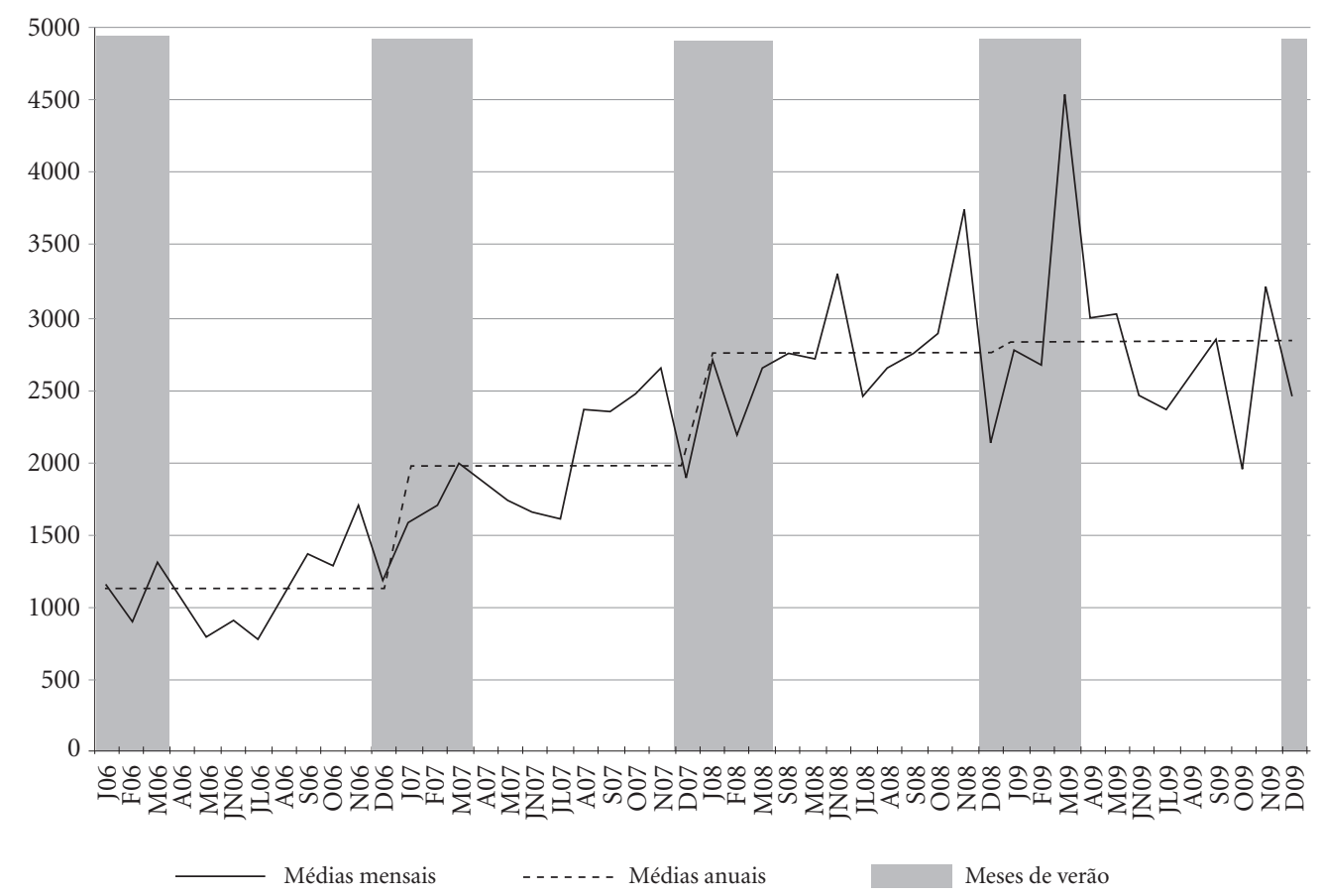

Gráfico 1. Evolução das Médias Mensais, médias anuais e período dos verões - acessos às páginas sobre prevenção de 2006 a 2009.

Tabela 1. Médias dos acessos anuais e nos verões dos conteúdos sobre prevenção do site do INCA.

\begin{tabular}{|c|c|c|c|c|c|c|c|c|}
\hline \multirow{3}{*}{ Ano } & \multicolumn{8}{|c|}{ Período de Análise } \\
\hline & \multicolumn{4}{|c|}{ Verão } & \multicolumn{4}{|c|}{ Ano completo } \\
\hline & Média & Desvio-Padrão & IC95\%* & Valor de $\mathrm{p}^{* *}$ & Média & Desvio-Padrão & IC95\%* & Valor de $\mathrm{p}^{* *}$ \\
\hline 2006 & 1141 & 165 & $(968-1314)$ & 0,7491 & 1141 & 165 & $(968-1314)$ & 0,7491 \\
\hline 2007 & 1799 & 178 & $(1612-1986)$ & & 1799 & 178 & $(1612-1986)$ & \\
\hline 2008 & 2426 & 282 & $(2130-2721)$ & & 2426 & 282 & $(2130-2721)$ & \\
\hline 2009 & 3102 & 951 & $(2106-4098)$ & & 3102 & 951 & $(2106-4098)$ & \\
\hline
\end{tabular}

*Intervalo de confiança para a média. ${ }^{* *}$ Valor de p obtido por meio da ANOVA.

ao sol. Robertson e Minkler observaram que 38\% dos banhistas americanos estavam plenamente conscientes dos riscos de câncer de pele, mas não tinham a intenção de usar filtros de proteção. Outros autores demonstram que os hábitos de prevenção também não são mais frequentes entre os sobreviventes de cânceres de pele mais graves (melanomas) quando comparados à população geral ${ }^{15}$.

É possível que os resultados do presente trabalho se justifiquem no cenário nacional pelo conhecimento insuficiente acerca das vulnerabi- lidades da saúde da pele, assim como das medidas ligadas à sua preservação. Por outro lado, tal indiferença coletiva talvez se associe à "cultura da medicação" ou ao preço dos filtros de proteção solar, inacessíveis a boa parte da população brasileira. Não obstante a tais ponderações, há autores que identificam nos segmentos sociais mais desfavorecidos uma noção de inefável superexposição a riscos onipresentes ${ }^{16}$, articulada à enunciação midiática de perigos decorrentes de fatores ainda pouco esclarecidos ao senso comum ${ }^{17}$. Seja por validação científica não devidamente com- 
provada ou por compreensão pública ainda precária, a reação dos banhistas americanos ${ }^{14}$ talvez possa ser atribuída ao "efeito avestruz" ligado à ideia de que submergimos em um mar de carcinogênicos contra o qual nenhuma proteção é suficiente $\mathrm{e}^{16,18}$. No contexto brasileiro, talvez o risco carcinogênico ligado a determinados fatores ainda careça de potência estruturante suficiente ao alinhamento das decisões cotidianas às pres- crições dos especialistas. A depender do ponto de observação e dos modelos explicativos dos quais nos valemos, muitas interrogações persistem em um terreno mutável ainda sujeito a muitos paradoxos $^{18-20}$. Certamente essas interrogações justificariam estudos posteriores, que articulem táticas de identificação de padrões de acessos a pesquisas socioantropológicas que teriam a acrescentar sentidos mais esclarecedores nesse campo.

\section{Colaboradores}

PR Vasconcellos-Silva trabalhou na concepção, nas análises e na redação final; RH Griep e MC de Souza trabalharam nas análises e na redação final.

\section{Agradecimentos}

Ao $\mathrm{CNPq}$ e à CAPES pelo financiamento do projeto. 


\section{Referências}

1. Smith BJ, Ferguson C, McKenzie J, Bauman A, Vita P. Impacts from repeated mass media campaigns to promote sun protection in Australia. Health Promot Int. 2002; 17(1):51-60.

2. Nutbeam D, Wise M, Bauman A, Harris E, Leeder S. National Goals and Targets for Australia's Health in the Year 2000 and Beyond. Sydney: Commonwealth Department of Health, Housing and Community Services; 1993.

3. Parkin DM, Whelan SL, Ferlay J, Teppo L, Thomas DB. Cancer Incidence in five countries. Lyon: International Agency for Research on Cancer; 1997. Vol VII (IARC Scientific Publications No. 143).

4. Coleman MP, Esteve J, Damiecki P, Arslan A, Renard H. Trends in Cancer Incidence and Mortality. Lyon: International Agency for Research on Cancer; 1993. (IARC Scientific Publications, no 121).

5. Vasconcellos-Silva PR, Castiel LD, Griep RH, Zanchetta M. Cancer prevention campaigns and Internet access: promoting health or disease? J Epidemiol Community Health 2008; 62(10):876-881.

6. Bernardo TM, Rajic A, Young I, Robiadek K, Pham MT, Funk JA. Scoping review on search queries and social media for disease surveillance: a chronology of innovation. J Med Internet Res 2013; 15(7):e147.

7. Ginsberg J, Mohebbi MH, Patel RS, Brammer L, Smolinski MS, Brilliant L. Detecting influenza epidemics using search engine query data. Nature 2009; 457(7232):1012-1014.

8. Ocampo AJ, Chunara R, Brownstein JS. Using search queries for malaria surveillance, Thailand. Malar $J$ 2013; 12(1):390.

9. Yom-Tov E, Gabrilovich E. Postmarket drug surveillance without trial costs: discovery of adverse drug reactions through large-scale analysis of web search queries. J Med Internet Res 2013; 15(6):e124.

10. Andersson T, Bjelkmar P, Hulth A, Lindh J, Stenmark S, Widerström M. Syndromic surveillance for local outbreak detection and awareness: evaluating outbreak signals of acute gastroenteritis in telephone triage, web-based queries and over-the-counter pharmacy sales. Epidemiol Infect 2014; 142(2):303-313.
11. Vasconcellos-Silva PR, Castiel LD, Rivera FJ. Assessing an Internet health information site by using log analysis: the experience of the National Cancer Institute of Brazil. Rev Panam Salud Publica 2003; 14(2):134-137.

12. Sociedade Brasileira de Dermatologia. Cuidados com a pele no verão. [acessado 2014 nov 20]. Disponível em: http://www.sbd.org.br/cuidados/cuidados-com-a-pele-no-verao/

13. Tjora A, Tran T, Faxvaag A. Privacy vs usability: a qualitative exploration of patients' experiences with secure Internet communication with their general practitioner. J Med Internet Res 2005; 7(2):e15.

14. Robertson A, Minkler M. New health promotion movements: A critical examination. Health Educ Quartely 1994; 21(3):285-312.

15. Mujumdar UJ, Hay JL, Monroe-Hinds YC, Hummer AJ, Begg CB, Wilcox HB, Oliveria SA, Berwick M. Sun protection and skin self-examination in melanoma survivors. Psychooncology 2009; 18(10):1106-1115.

16. Kreuter M. Human behaviour and cancer: forget the magic bullet. Cancer 1993; 72(Supl. 3):996-1001.

17. Baillie L, Basset-Smith J, Broughton S. Using communicative action in the primary prevention of cancer. Health Educ Behav 2000; 27(4):442-453.

18. Evans R, Barer M, Marmor T, editors. Why are some people healthy and others not? The determinants of health of populations. New York: Aldine; 1994.

19. Lupton D. Communicating health: the mass media and advertising in health promotion. In: Lupton D. The imperative of health. Public health and the regulated body. Londres: Sage; 1995. p. 148-157.

20. Lupton D. The emotional self: a sociocultural exploration. London: Sage; 1998.

Artigo apresentado em 15/08/2014

Aprovado em 04/12/2014

Versão final apresentada em 06/12/2014 